\title{
Peripheral functional organisation of vagally evoked gastric motor responses in the ferret
}

\author{
P L R ANDREWS, I N C LAWES* AND A J BOWER \\ From the Department of Physiology and the Department of Human Biology and Anatomy, \\ University of Sheffield, Sheffield
}

SUMMARY The aims of the present study were to determine the relative amplitudes of intragastric motor responses evoked by different vagal branches and to establish whether the effects of acute or chronic vagotomy could be predicted from these data. Intragastric pressure responses to electrical stimulation of the vagus were measured in urethane-anaesthetised ferrets and acute or chronic vagotomies were performed. The results show that the left and right cervical vagi were equipotential and fully overlapped each other. Their contributions to the dorsal trunk were equipotential and fully overlapping and so were their contributions to the ventral trunk. The dorsal trunk was more effective than the ventral trunk and there was total functional overlap between these two trunks. Vagal evoked gastric motor responses of the ferret are apparently organised in a different way from vagally induced acid secretion or hormone release in the cat. Acute removal of a trunk led to a reduction in evoked responses that was not a linear function of the effect of stimulation of that trunk. In contrast, chronic removal caused a relative increase in evoked responses that was inversely related to the decrease caused by acute removal. The implications of total functional overlap and neuromuscular reorganisation after chronic vagotomy are discussed.

The majority of papers concerning vagal influence on gastric functions usually fail to mention which cervical or abdominal vagal trunk was stimulated, apparently based on the assumption that the vagi are equipotential. However, a recent report by Bauer ${ }^{1}$ demonstrated that the left cervical vagus of the cat evoked a greater secretion of gastric juice than the right. It has been reported that each cervical vagus induced the release of a similar quantity of gastrin ${ }^{2}$ and the vagi were additive in effect; a similar pattern has been demonstrated for the vagal release of insulin ${ }^{3}$ from the pancreas.

The aim of the present study was to examine whether vagal evoked motor responses of the stomach were organised in a similar way to the regulation of hormone release and acid secretion. At the same time the effects of acute and chronic vagotomy on gastric motor response to well-defined stimuli were compared in order to establish whether the effect of removal of a vagal branch could be predicted from

*Address for correspondence: I N C Lawes, Department of Human Biology and Anatomy, University of Sheffield, Western Bank, Sheffield S10 2TN.

Received for publication 13 May 1980 the response to electrical stimulation of that branch.

By using the intragastric pressure response to a standard stimulus it was hoped to obtain less variable results than might be obtained from observation of the gross electrical activity of gastric smooth muscle, ${ }^{4}$ changes in the interdigestive myoelectric complex ${ }^{5}$ or gastric emptying time. ${ }^{6}$

Ferrets were used in this study in order to relate to extensive investigations of afferent ${ }^{7}$ and efferent ${ }^{89}$ traffic in the vagus and to previous work on vagal regulation of gastric secretory ${ }^{1011}$ and motor activity. ${ }^{12}$

\section{Method}

Fifty-four ferrets weighing between $400 \mathrm{~g}$ and $1600 \mathrm{~g}$ were used. They were fed on a standard carnivore diet with free access to water. Thirty-six animals were anaesthetised with an intraperitoneal injection of pentobarbitone sodium (May \& Baker Ltd.) $\left(60 \mathrm{mg} \mathrm{kg}^{-1}\right)$ and subjected to left cervical, right cervical, subdiaphragmatic dorsal, or ventral vagotomy (Fig. 1). Under aseptic conditions the appropriate nerve was mobilised and a section $5 \mathrm{~mm}$ long removed. The wound was then closed in layers. 


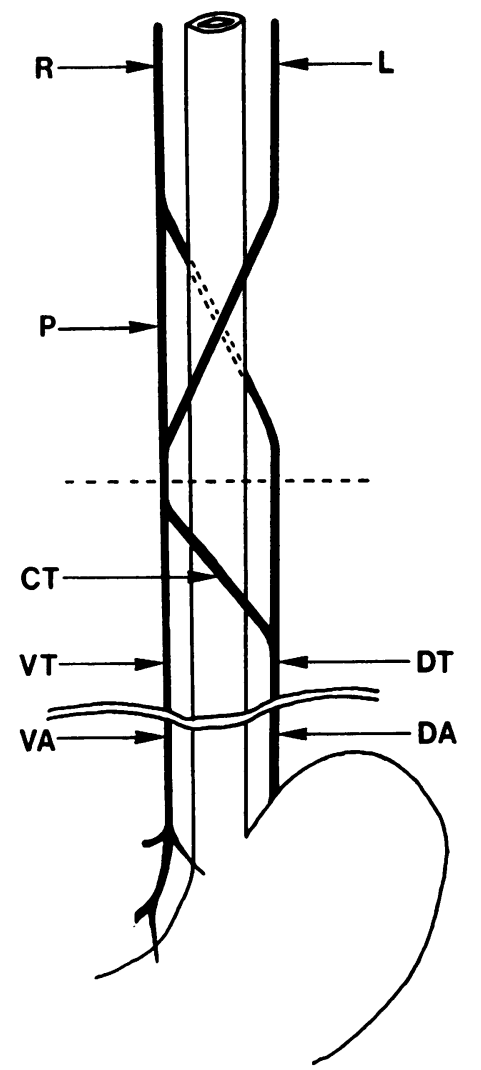

Fig. 1 A simplified schematic drawing showing the organisation of the cervical and abdominal vagi. The vagus was stimulated immediately distal to $R, L, C T$, $V T$, and DT. Acute vagotomies were performed at $R, L, C T, V T$, and DT. Chronic vagotomies were performed at $R, L, V A$, and $D A$. $R$ : right cervical vagus; $L$ : left cervical vagus; $P$ : plexus. At this point a plexus is seen carrying fibres from the right cervical vagus to the left thoracic trunk; CT: crossing branch; $V T$ : ventral (thoracic) branch; DT: dorsal (thoracic) branch; $V A$ ventral (abdominal) branch; $D A$ : dorsal (abdominal) branch.

These animals were restricted to a diet of water on the first postoperative day, milk on the second day, and dog meat or dead rats with fluid ad libitum thereafter. Animals with cervical vagotomy displayed ipsilateral ptosis indicating the intimate relationship between the vagus and cervical sympathetic trunk in the ferret. Up to $20 \%$ of the body weight was lost postoperatively. After 14 days these animals were prepared in the same way as the remaining 18 animals as described below.

Food was withheld for 24 hours before experimentation. Anaesthesia was achieved with an intraperitoneal dose of urethane $(1.5 \mathrm{~g} / \mathrm{kg}, 50 \%$ in $0.9 \%$ $\mathrm{NaCl}$ ); supplementary doses of $25 \%$ urethane were given intravenously if required. A wide bore cannula was inserted into the trachea via a tracheostomy and the right external jugular vein was cannulated to provide a route for subsequent doses of anaesthetic. The stomach was intubated via the mouth with a plastic open-ended tube and maintained in position with a ligature around the cervical oesophagus. The abdominal viscera were exposed by a mid-line incision and the pylorus was occluded by an external ligature. The abdominal incision was then closed in layers. The vagi were exposed in the neck and separated from the carotid arteries for a distance of $1 \mathrm{~cm}$, gently cleared of connective tissue and sectioned as far rostrally as possible. The vagal branches in the thorax coalesce about $2-3 \mathrm{~cm}$ above the diaphragm to form dorsal and ventral trunks which travel on the surface of the oesophagus. There is a consistent branch in the thorax which travels on the left surface of the oesophagus, caudal to the heart, apparently linking the left thoracic vagus to the right thoracic vagus. We have called this the crossing branch (Fig. 1). The ventral trunk was taken as being distal to the point at which the crossing branch leaves the left thoracic vagal trunk and the dorsal trunk as being distal to the point at which the crossing branch joins the right thoracic vagal trunk. The thoracic vagal trunks just rostral to the diaphragm were exposed by a left thoracotomy and removal of the 11 th and 12 th ribs. The dorsal and ventral trunks, and the crossing branch were mobilised just sufficiently to allow them to be placed over small silver or platinum stimulating electrodes. After the thoracotomy the animals were ventilated with room air by an intermittent positive pressure system. An end expiratory resistance of $3 \mathrm{~cm} \mathrm{H}_{2} \mathrm{O}$ was used. The rectal temperature was maintained at $39^{\circ} \mathrm{C}$ by means of radiant heat or a homeothermic electric blanket.

\section{NERVE STIMULATION}

The nerves were placed over bipolar silver or platinum electrodes. Stimuli of $0.5 \mathrm{~ms}$ width, 20-30 V were delivered from a Devices isolated stimulator. The pulses were precisely timed with a Digitimer D4030. Periods of stimulation of 10 seconds at $10 \mathrm{~Hz}$ were used, as these parameters produce a single gastric contraction of maximal amplitude. An interstimulus interval of three minutes was used in order to obtain the maximal amplitude of contraction from each stimulation and to allow the intragastric pressure to reach a stable level before the next stimulation. The nerves were bathed in warm liquid paraffin and the electrodes moved caudally at intervals to prevent damage to the nerve by prolonged stimulation at one site. The sequence of stimulation of the different branches 
was varied to prevent order effects. Three stimuli at each site were used, except where a sequence of stimulation was given to obtain traces for illustration.

\section{INTRAGASTRIC PRESSURE RECORDING AND ANALYSIS}

Twenty millilitres of $0.9 \%$ saline at $37^{\circ} \mathrm{C}$ was introduced into the stomach in order to provide a constant volume system from which intragastric pressure could be recorded. This was done by attaching the end of the oesophageal intragastric tube to a fluid pressure transducer (SEM 4-88, SE Labs, Ltd.). The transducer signal was amplified (EMMA, SE Labs Ltd.) and displayed on a Bryans 28000 chart recorder. The amplitude of the evoked changes in the intragastric pressure was measured in $\mathrm{cm} \mathrm{H}_{2} \mathrm{O}$ above the resting pressure.

PRE-STIMULUS VAGOTOMIES

When all five sites - that is, the two cervical vagi, the ventral and dorsal vagi, and the crossing branch - had been stimulated and the evoked increase in intragastric pressure recorded, the dorsal trunk, ventral trunk, or crossing branch was cut. The remaining intact branches were then restimulated and the evoked intragastric pressure recorded.

The sites of the chronic vagotomies were confirmed by dissection post mortem.

\section{STATISTICS}

In order to compare the different sites of stimulation and vagotomy, a two-way analysis of variance was carried out on the results from each group of animals and a one-way analysis of variance was carried out on the results from all groups of animals. Except for the group which received an acute ventral vagotomy, all the variance ratios were significant at the $5 \%$ level or better. Comparisons were then made using Student's $t$ test. Paired sample $t$ tests were used for results from within the same group of animals, and unpaired $t$ tests when the results were from different groups of animals. All significant results are at the $5 \%$ level or better.

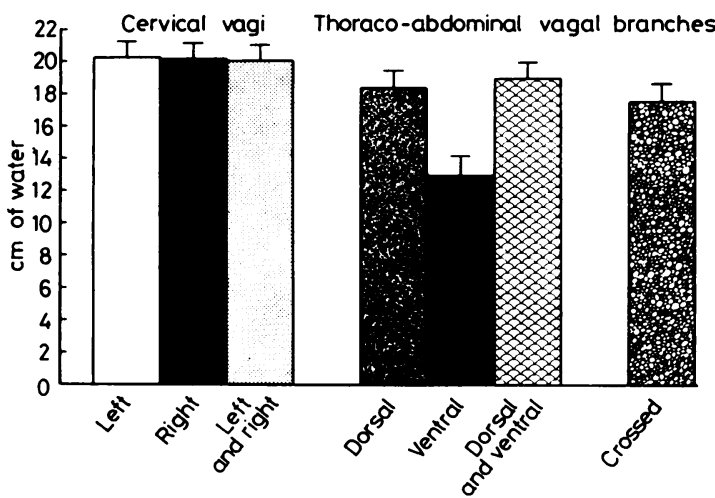

Fig. 2 Mean and standard errors of the intragastric pressure response to stimulation of the vagus nerve at the sites indicated in Fig. I. The results are from 18 ferrets before acute thoracic vagotomy.

\section{Results}

Stimulation of the vagi evoked a single gastric contraction and the ensuing rise in intragastric pressure was recorded. The amplitude of the change in intragastric pressure depended on the site of stimulation, as shown for the 18 acutely prepared animals before vagotomy (Fig. 2). It depended also on which branches were vagotomised and whether the vagotomy was acute (Table 1) or chronic (Table 2 ). Results are discussed in terms of relative and absolute amplitudes of response before vagotomy and after acute or chronic vagotomy.

\section{CONTROL RESPONSES}

\section{Absolute amplitudes}

The responses to stimulation in 18 acutely prepared ferrets are shown in Fig. 2. Within any animal the responses were consistent and reproducible (Fig. 3).

\section{Relative amplitudes}

Statistical analysis confirmed the impression to be gained from inspection of Fig. 2. The left and right

Table 1 Effects of acute vagotomy

\begin{tabular}{|c|c|c|c|}
\hline \multirow[t]{2}{*}{ Site of stimulation } & \multicolumn{2}{|c|}{ Changes in amplitude resulting from: } & \multirow[b]{2}{*}{ Crossing vagotomy } \\
\hline & Dorsal vagotomy & Ventral vagotomy & \\
\hline $\begin{array}{l}\text { Left cervical } \\
\text { Right cervical } \\
\text { Left with right } \\
\text { Dorsal trunk } \\
\text { Ventral trunk } \\
\text { Dorsal with ventral } \\
\text { Crossing branch }\end{array}$ & $\begin{array}{l}-8 \cdot 7 \pm 2 \cdot 8 * \\
-9 \cdot 0 \pm 2 \cdot 7 \dagger \\
-8 \cdot 6 \pm 2 \cdot 7 * \\
-0 \cdot 6 \pm 1 \cdot 0 \\
-18 \cdot 0 \div 1 \cdot 6\end{array}$ & $\begin{array}{l}-2.6 \pm 1.0^{*} \\
-3.9 \pm 0.5 \ddagger \\
-1.8 \pm 0.7^{*} \\
-1.2 \pm 0.6 \\
- \\
-0.7 \pm 0.8\end{array}$ & $\begin{array}{l}-9.6 \pm 2.6 \dagger \\
-1.5 \pm 0.7 \\
-1.4 \pm 0.8 \\
-0.4 \pm 1.0 \\
-0.3 \pm 0.9 \\
-1.4+0.8\end{array}$ \\
\hline
\end{tabular}

Mean and standard errors of the change in amplitude of intragastric pressure responses brought about by acute dorsal, ventral, or crossing branch vagotomy. The sites of stimulation and vagatomy are shown in Fig. 1. There were six animals in each group. Significance levels from paired sample $t$ tests are shown by: $* \mathbf{P}<0.05 ;+\mathrm{P}<0.02 ; \ddagger \mathrm{P}<0.001$. 
Table 2 Effects of chronic vagotomy

\begin{tabular}{|c|c|c|c|c|}
\hline \multirow[t]{2}{*}{ Site of stimulation } & \multicolumn{3}{|c|}{ Changes in amplitude brought abut by: } & \multirow[b]{2}{*}{$\begin{array}{l}\text { Ventral abdominal } \\
\text { vagotomy }\end{array}$} \\
\hline & $\begin{array}{l}\text { Left cervical } \\
\text { vagotomy }\end{array}$ & $\begin{array}{l}\text { Right cervical } \\
\text { vagotomy }\end{array}$ & $\begin{array}{l}\text { Dorsal abdominal } \\
\text { vagotomy }\end{array}$ & \\
\hline Left cervical & - & $-1 \cdot 5: 1 \cdot 5$ & $6.6: 2.5^{*}$ & $2.4: 2.8$ \\
\hline Right cervical & $5.6 \div 1.8$ & - & $9 \cdot 6: 2 \cdot 7 \ddagger$ & $5 \cdot 0 \div 2 \cdot 5$ \\
\hline Left with right & - & - & $7.2: 2.6 \dagger$ & $5 \cdot 2 \cdot 2.9$ \\
\hline Dorsal trunk & $.4 .8 \div 1.8 \dagger$ & $-0.9: 2.0$ & $\ldots$ & $4 \cdot 3: 2 \cdot 5$ \\
\hline Ventral trunk & $3 \cdot 5 \div 2 \cdot 3$ & $-1 \cdot 2: 1 \cdot 8$ & $-5 \cdot 5: 2.3 *$ & - \\
\hline Dorsal with ventral & $+5 \cdot 5 \pm 1 \cdot 9$ & $-0.6: 1.8$ & - & - \\
\hline Crossing branch & $-6 \cdot 1 \pm 2 \cdot 2 \ddagger$ & $-1 \cdot 0: 1 \cdot 5$ & $0 \cdot 0-0.0$ & $5 \cdot 1 \div 2 \cdot 6$ \\
\hline
\end{tabular}

Mean and standard errors of the difference in amplitude between acute and chronic post-vagotomy intragastric responses. The sites of stimulition and vagotomy are shown in Fig. 1. The difierences are displayed as amplitudes after chronic vagotomy minus amplitudes after acute vagotomy. Signifizance levels from unpaired sample $t$ tests are shown by: ${ }^{*} \mathbf{P}<0.05 ; \dagger \mathbf{P}<0.02 ; \dot{+}<00.01$.

cervical vagi had an equal effect on evoked intragastric pressure, and the effect of stimulating both together was the same as stimulating either alone.

The dorsal trunk had a greater effect than the

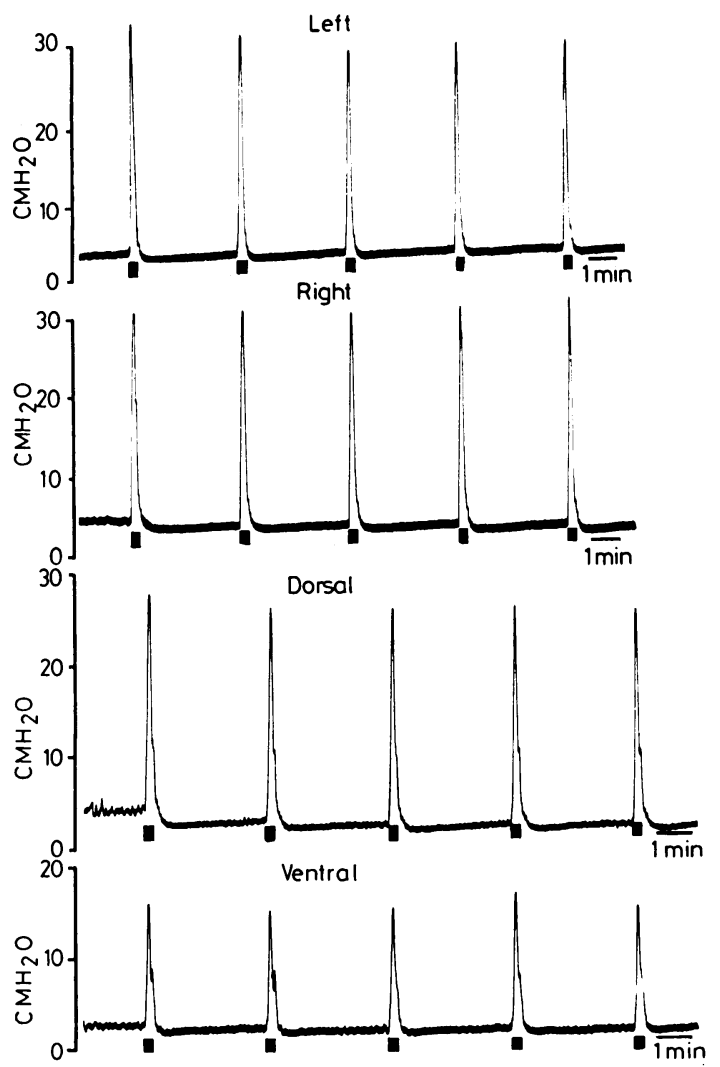

Fig. 3 The upper pair of traces shows the rise in intragastric pressure seen when the left or right cervical vagi are stimulated at the parameters in the text. The lower pair of tracings show the result of stimulating the dorsal or ventral trunks at the same parameters. The small bars indicate the periods of stimulation (10) seconds). ventral trunk $(\mathbf{P}<0.002)$. The effect of stimulating both trunks simultaneously was the same as stimulating the dorsal trunk alone, but significantly greater than stimulation of the ventral trunk alone $(\mathrm{P}<0.001)$. Cervical stimulation was marginally but significantly more effective than thoracic stimulation $(\mathrm{P}<0.05)$.

\section{ACUTE VAGOTOMY} Absolute amplitudes

As shown in Table 1 and confirmed by statistical analysis, dorsal vagotomy produced large and significant decreases in response to cervical stimulation $(P<0.05)$. The response to stimulation of the crossing branch was abolished.

Ventral vagotomy produced small but significant decreases in responses to cervical stimulation $(\mathrm{P}<0.05)$ but had no effect on the crossing branch responses.

Cutting of the crossing branch produced a large reduction in the response to stimulation of the left cervical vagus $(P<0.02)$ but it had no other significant effects.

Neither truncal vagotomy had any effect on the response to stimulation of the other trunk (dorsal or ventral).

\section{Relative amplitudes}

Neither ventral nor dorsal vagotomy altered the relationship between responses to cervical stimulation: left, right, and bilateral cervical stimulation evoked responses of statistically similar amplitude.

After crossing branch vagotomy, however, the left cervical branch was significantly less effective than the right cervical branch $(\mathrm{P}<0.05)$.

\section{CHRONIC VAGOTOMY \\ Absolute amplitudes}

The amplitude of responses to stimulation of surviving branches after chronic vagotomy was compared to the corresponding amplitudes obtained 
from acutely prepared animals. The difference between the two is shown in Table 2.

After chronic left cervical vagotomy the response to stimulation of the crossing branch was greatly reduced $(\mathrm{P}<0.01)$. All other responses were increased, the increase reaching significant levels $(\mathrm{P}<0.02)$ in all cases except after ventral stimulation.

Chronic right vagotomy had no significant effect on any of the responses.

Relative to the effect of acute dorsal vagotomy, responses to stimulation after chronic dorsal vagotomy were all significantly larger $(P<0.05)$, except in the case of the crossing branch. Stimulation of the latter produced no response after either acute or chronic vagotomy.

Chronic ventral vagotomy also resulted in larger responses than were obtained after acute ventral vagotomy, but none of the differences reached significant levels.

\section{Relative amplitudes}

The relative amplitudes of response to left, right, or bilateral cervical stimulation were unchanged by chronic abdominal vagotomy-none of the differences between them was statistically significant.

After chronic cervical vagotomy the dorsal trunk was significantly more effective than the ventral trunk $(P<0.05)$. Simultaneous stimulation of both trunks was not significantly different from stimulation of the dorsal trunk alone.

\section{Mortality associated with chronic vagotomy}

Mortality was analysed in terms of its relationship to body weight, sex, and site of vagotomy. Animals with a body weight less than $1100 \mathrm{~g}$ had two and a half times the mortality of heavier animals. There was no correlation with sex provided the animals were matched for weight. The mortality rate after ventral vagotomy was twice that for other types of vagotomy.

\section{Discussion}

When we pool these results, several conclusions may be drawn. Stimulation of left or right cervical vagi gave equal responses even after dorsal or ventral vagotomy, therefore the cervical vagi must make a functionally equal efferent contribution within each abdominal trunk. This contrasts with the assertion that, in man, the ventral trunk is derived mainly from the left cervical vagus while the dorsal trunk is derived mainly from the right cervical vagus. ${ }^{14}$ Our physiological results are in agreement with histological studies on rabbit ${ }^{14}$ and dog. ${ }^{15}$ It is therefore legitimate to ask if the assertion concerning the arrangement in man is a genuine species difference.

One of the main conclusions to be drawn is that there is considerable redundancy in the vagal effects on gastric motor responses. The left and right cervical vagi showed total functional overlap, both before and after single truncal vagotomy. Furthermore, the ventral trunk is completely overlapped in its motor effects by the dorsal trunk. In other species (cat) lack of overlap, and even addition, has been shown for vagally evoked production of gastric acid, ${ }^{1}$ gastrin, ${ }^{2}$ and insulin. ${ }^{3}$ While this may represent a species difference, Kewenter ${ }^{16}$ commented that in the cat there was overlap between the vagi in their effects on motility in the ileum. It is therefore possible that the secretory and motor functions of the vagus may be organised in a fundamentally different way.

Our results do not indicate the mechanism of this total functional overlap. Anatomical convergence of excitatory or inhibitory fibres on post-ganglionic neurons or on smooth muscle cells, or electrical coupling between independently innervated pools of smooth muscle are possibilities. In view of this uncertainty, and because of the double overlap first of cervical effects and then again of abdominal effects, it is premature to attempt to define a model of the relative contributions to gastric motor activity of each vagal branch.

A major finding in this study is the contrast between the effects of acute and chronic vagotomies. Acute dorsal vagotomy produced a decrease in response amplitude of $41 \%$ and acute ventral vagotomy produced a decrease in response amplitude of $9 \%$. While these reductions are not commensurate with the responses to stimulation of these trunks $(96 \%$ of the maximum available response for the dorsal trunk, $68 \%$ for the ventral trunk), they are a logical consequence of the degree of functional overlap between the two trunks. By contrast, responses after chronic dorsal vagotomy were $57 \%$ larger than after acute dorsal vagotomy. Responses after chronic ventral vagotomy were $30 \%$ larger than after acute ventral vagotomy. Chronic vagotomy appears to provoke neuromuscular reorganisation of a kind that allows the surviving vagal branches to become considerably more effective.

It is to be noted that stimulation of the dorsal trunk gave larger responses than stimulation of the ventral trunk, acute dorsal vagotomy caused greater decrements in cervical responses than acute ventral vagotomy, and chronic dorsal vagotomy revealed a greater relative recovery than chronic ventral vagotomy. If the potency of a branch reflects the number of axons and terminals, then dorsal vagotomy should result in more extensive degeneration 
than ventral vagotomy. If the degeneration is the critical stimulus for neuromuscular reorganisation then it follows that dorsal vagotomy should also result in a more extensive neuromuscular reorganisation than ventral vagotomy.

Providing that these two assumptions are accepted -that is, the equation of motor efficacy with numbers of nerve terminals, and degeneration of terminals as a stimulus for neuromuscular reorganisation-the apparent disparity between the effects of acute and chronic vagotomy is resolved for abdominal trunks. The asymmetry of increases caused by chronic cervical vagotomy do not relate to the symmetry of evoked responses in acute preparations, however. Schofield ${ }^{17}$ working on rats found that left cervical vagotomy increased the number of argyrophilic swellings found in the gastric wall while right cervical vagotomy did not. If these findings apply to the ferret then left cervical vagotomy would produce more degeneration than right cervical vagotomy and on the previous assumptions this would therefore account for the greater increase in evoked motor activity after chronic left vagotomy.

As with functional overlap, the mechanism of neuromuscular reorganisation is not evident from our results. Among the possibilities are collateral sprouting from surviving branches as seen in the superior cervical ganglion; ${ }^{18}$ unmasking of synapses $;^{18}$ increased sensitivity of postganglionic neuronal membrane to neurotransmitters; ${ }^{20}$ changes in the levels of acetylcholinesterase. ${ }^{21}$ The simultaneous existence of total functional overlap and increased effectiveness of surviving vagal branches after chronic vagotomy presents a paradox whose resolution would be of considerable interest.

Total functional overlap and marked compensation (even overcompensation) after chronic vagotomy should both prevent any major disruption of gastric motor activity after single truncal vagotomy in ferrets. Unless the disruption of gastric motility seen in man $^{22}$ is a species difference, it may be that the disruption is not so much loss of the vagal ability to modulate gastric contractions, but more a disorganisation of vagal efferent discharge secondary to a loss of afferent information. ${ }^{23}$

The implications of this work are clear. First, because of total functional overlap (or redundancy) and the inverse relationship between the effects of acute and chronic vagotomy (plasticity), caution must be exercised in the extrapolation of the results of acute vagotomies to clinical situations. Secondly, the changes in neuromuscular organisation of the stomach seen here may provide some insight into the problems of long-term changes after clinical vagotomy.

\section{References}

${ }^{1}$ Bauer RF. Electrically induced vagal stimulation of gastric acid secretion. In: Nerves and the gut. Brooks FP, Evers PW, eds, New Jersey, CB Slack Inc, 1977: 86-95.

${ }^{2}$ Uvn̈as-Wallensten K, Uvn̈as B, Nilsson G. Quantitative aspects of the vagal control of gastrin release in cats. Acta Physiol Scand 1976; 96: 19-28.

${ }^{3}$ Uvñas-Wallensten K, Nilsson G. A quantitative study of the insulin release induced by vagal stimulation in anesthetised cats. Acta Physiol Scand 1978; 112: 137-42.

${ }^{4}$ Kelly KA, Code CF. Effect of transthoracic vagotomy on canine gastric electrical activity. Gastroenterology 1969;57: 51-8.

${ }^{5}$ Ruckebusch Y, Bueno L. Migrating myoelectrical complex of the small intestine. Gastroenterology 1977; 73: 1309-14.

${ }^{6}$ Wilbur BG, Kelly KA. Effect of proximal gastric, complete gastric, and truncal vagotomy on canine gastric electrical activity, motility, and emptying. Ann Surg 1973; 178: 295-303.

${ }^{7}$ Andrews PLR, Grundy D, Scratcherd T. Vagal afferent discharge from mechano-receptors in different regions of the ferret stomach. J Physiol (Lond) 1980; 298: 513-24.

${ }^{8}$ Andrews PLR, Salih AA, Scratcherd T. Modulation of single vagal efferent fibre discharge by gastric afferents in the ferret (abstract). $J$ Physiol (Lond) 1978; 284: 40p.

${ }^{9}$ Andrews PLR. The vagal control of the gastrointestinal tract. PhD thesis 1979. University of Sheffield.

${ }^{10}$ Basso N, Umeda N, Roth JLA, Passaro E Jr, Pfeiffer CJ. Effect of unilateral vagotomy on gastric secretions in the ferret. Gastroenterology 1971 ; 61 : 207-12.

${ }^{11}$ Andrews PLR, Bower AJ. Simultaneous measurement of gastric secretion and motility in response to various stimuli in the ferret (abstract). J Physiol (Lond) 1977; 275: 53p.

${ }^{12}$ Andrews PLR, Grundy D, Scratcherd T. Reflex excitation of antral motility induced by gastric distension in the ferret. J Physiol (Lond) 1980; 298 : 79-84.

${ }^{13}$ Warwick R, Williams PL eds. Gray's anatomy 35th ed Edinburgh: Longman, 1973: 1275.

${ }^{14}$ Evans DHL, Murray JG. Histological and functional studies on the fibre composition of the vagus nerve of the rabbit. $J$ Anat $1954 ; 88$ : 320-37.

${ }^{15}$ Kemp DR. A histological and functional study of the gastric mucosal innervation of the dog. I. the quantification of the fibre content of the normal supra-diaphragmatic vagal trunks and their abdominal branches. Aust NZ J Surg 1973; 43: 288-94.

${ }^{16} \mathrm{Kewenter} \mathrm{J}$. The vagal control of the jejunal and ileal motility and blood flow. Acta Physiol Scand 1965; 65: suppl 251.

${ }^{17}$ Schofield GC. Experimental studies on the myenteric plexus in mammals. J Comp Neurol 1962; 119: 159-85.

${ }^{18}$ Murray JG, Thompson JW. The occurrence and function of collateral sprouting in the sympathetic nervous system of the cat. J Physiol (Lond) 1957; 135: 133-62.

${ }^{10}$ Cass DT, Sutton TJ, Mark RF. Competition between nerves for functional connections with axolotl muscles. Nature 1973; 243: 201-3. 
${ }^{20} \mathrm{Kuffler} \mathrm{SW}$, Dennis MJ, Harris AJ. The development of chemosensitivity in extrasynaptic areas of the neuronal surface after denervation of parasympathetic ganglion cells in the heart of the frog. Proc Royal Soc Series B 1971 ; 177 : 555-63.

${ }^{21}$ Schmid W, Van der Zypen E, Keller H. Die Wirkung einer subtotalen Vagotomie auf den Plexus myentericus
(Auerbach) verschiedener Darmabschnitte. Acta Anat (Basel) 1979; 104: 36-51.

${ }^{22}$ Duthie HL, Wormsley KG. eds. Scientific basis of gastroenterology. Edinburgh ,Churchill Livingstone, 1979.

${ }^{23}$ Davison JS, Grundy D. Modulation of single vagal efferent fibre discharge by gastrointestinal afferents in the rat. $J$ Physiol (Lond) 1978; 284: 69-82. 